\title{
The effects of astaxanthin supplementation on the expression of miR-146a and miR-126, oxidative stress and inflammation in patients with type 2 diabetes mellitus: A randomized double blind placebo controlled clinical trial
}

\author{
Amirhossein farokhzad ${ }^{1}$, Nafiseh Sokri Mashhadi ${ }^{2}$, Maryam Tahmasebi ${ }^{3}$, Javad \\ Mohammadi-asl ${ }^{3}$, Mehrnoosh Zakerkish ${ }^{3}$, and Majid Mohammadshahi ${ }^{3}$ \\ ${ }^{1}$ Affiliation not available \\ ${ }^{2}$ Isfahan University of Medical Sciences \\ ${ }^{3}$ Ahvaz Jondishapour University of Medical Sciences
}

September 16, 2020

\begin{abstract}
Background: The pathogenesis of type 2 diabetes (T2D) is associated with chronic oxidative stress and inflammation. It is well known that the expression of some miRNAs such as miRNA-146a up- regulate in diabetic and hyperglycemic patients whereas, circulating miRNA-126 reduces. Therefore, the purpose of this study was to determinate the effects of Astaxanthin supplementation on the expression of miR-146a and miR-126, MDA and inflammation in patients with type 2 diabetes. Methods: This randomized double-blind placebo-controlled clinical trial was conducted on 44 type 2 diabetes patients who were randomly assigned to received $8 \mathrm{mg}$ of oral Astaxanthin/d $(n=22)$ or placebo( $n=22)$ for 8 weeks. Results: We observed that Astaxanthin supplementation could decrease plasma levels of MDA and IL-6 $(\mathrm{P}<0.05)$ and declined the expression level of miR-146a over time (Fold change: $-1 / 388)(\mathrm{P}<0.05)$. Conclusion: Astaxanthin supplementation might be beneficial for improving circulating MDA and IL-6 and down regulation of miR-146a. However, future investigations are suggested to confirm these results.
\end{abstract}

\section{What's known?}

Some previous studies showed the antioxidant and antinflamatory effects of astaxanthin.

There have been no human studies to investigate the effect of astaxanthin or its constituents on the expression of miR-146a and miR-126, oxidative stress and inflammation in patients with type 2 diabetes mellitus.

\section{What new}

This study is the first human investigation that was designed to evaluate the effect of astaxanthin or its constituents on the expression of miR-146a and miR-126, oxidative stress and inflammation in patients with type 2 diabetes mellitus.

The present study suggests that astaxanthin supplementation might be beneficial for improving circulating MDA and IL-6 and down regulation of miR-146a.

\section{Introduction}

Type 2 diabetes mellitus (T2DM) is a metabolic diseases recognized by hyperglycemia due to insulin resistance and insufficient insulin secretion ${ }^{1}$. Growing evidence indicated that chronic oxidative stress and inflammation closely involved in the pathogenesis of T2DM and the progression of related complications ${ }^{2,3}$. 
Systemic inflammation can affect $\beta$-cell functionality and promote mitochondrial dysfunction that results in precedes T2D manifestations and predicts its progression ${ }^{4}$.

MicroRNAs (miRNAs) are small noncoding RNA which are usually transcribed by RNA polymerase II ${ }^{5}$. They modulate the gene expression level through their interaction by 3'UTR of the genes which is eventually lead to RNA degradation ${ }^{6}$. The miRNAs expression is tissue-specific, but they may be observed in the circulation ${ }^{7}$. Previous studies detected the up regulation of some miRNAs such as miRNA-146a in diabetic and hyperglycemic patients whereas, circulating miRNA-126 are reduced ${ }^{8,9}$. In addition, participating of MicroRNAs in T2D pathogenesis is indicated through the decline of insulin release and an increase of pancreatic $\beta$-cell apoptosis ${ }^{10}$. So, from a clinical perspective, the levels of miR-26a and miR-146a can be evaluated in the circulation at different disease stages related to $\mathrm{T} 2 \mathrm{D}^{11}$.

Recently, there has been significant interest in the potential role of food antioxidants in controlling lipid peroxidation, diabetes-induced inflammation and modulating microRNAs ${ }^{12,13}$. Astaxanthin (AST) is a carotenoid belongs to the xanthophyll family and common pigment in algae and fish ${ }^{14}$. Recent studies demonstrated the antioxidant effect of Astaxanthin on in the treatment and prevention of diabetes ${ }^{15}$. However, few studies assessed the efficacy of AST on circulating miRNAs, and reducing inflammation and oxidative stress in diabetes patients. Therefore, this study was aimed to determinate the effects of AST supplementation on the expression of miR-146a and miR-126, MDA and inflammation in patients with type 2 diabetes.

\section{Materials and Methods}

\section{Population and Study design}

In the double-blind, randomized placebo-controlled trial manner, Forty-four volunteers with type 2 diabetes were joined to this study. These individuals referred to the Diabetes Research Center at the Ahvaz University of Medical Sciences and met our inclusion criteria including 30-60 years old subjects, definitive diagnosis of type II diabetes with no insulin therapy, no pregnancy and lactation, no self-reported specific diseases and malignancies, no kidney failure, no heart disease, no thyroid and other inflammatory disease, no vitamin and antioxidant supplement during the last 6 months, and no smoking and drinking. The written consent was obtained from each individual before joining to the project.

All processes were performed under the supervision of a nutritionist at each step, during which the participants were counseled to eat an exact diet, perform physical activity, and take blood glucose lowering drugs. Qualified researchers performed all the data collection and measurements. Basal characteristics were collected by the interviewers which is presented before for subjects $(n=22)$ or placebo $(n=22)$ for 8 weeks. In a double-blinded fashion, subjects received $8 \mathrm{mg}$ of oral Astaxanthin/d (nature vision; USA) which was derived from Haematococcus pluvialis (microalgae) and inactive ingredients which were used for both supplement and placebo were Dicalcium Phosphate, Microcrytalline Cellulose, Stearic Acid, Silicon Dioxide, Magnesium Stearate. Food consumption was recorded before, during and after the study period (3-day food records), according to volume or weight and type of food. To assess energy and macronutrient intake, the dietary data were analyzed using Nutritionist IV software (Version 4.1, First Databank Division, The Hearst Corporation, and San Bruno, CA, USA). During each 2-week appointment, the participants' compliance to the study protocol was assessed by numeration the residual AST tablets, and asking them to preserve their habitual diet, lifestyle, and medication ingestion.

\section{Blood Sampling and Primary Measures}

Venous blood samples were collected in the fasting state for biochemical measurements. Samples were centrifuged and serums were frozen and stored at $-80{ }^{\circ} \mathrm{C}$. The serum level of IL- 6 was determined using the method described by the Boster human IL-6 ELISA kit. Malondialdehyde (MDA) level was measured as an index of lipid peroxidation using TBRAS assay protocol. The MDA reacts with thiobarbituric acid (TBA) as a thiobarbituric acid reactive substance (TBARS) to produce a red colored complex. The absorbance of the samples was measured at 532nm using ELISA plate reader. $3 \mathrm{ml}$ phosphoric acid (1\%) and $1 \mathrm{ml}$ TBA 
$(0.6 \%)$ was added to $0.5 \mathrm{ml}$ of homogenate in a centrifuge tube and the mixture was heated for 45 min in a boiling water bath. After cooling, $4 \mathrm{ml}$ of n-butanol was added the mixture and vortex-mixed for $1 \mathrm{~min}$ followed by centrifugation at $20000 \mathrm{rpm}$ for $20 \mathrm{~min}$. The organic layer was transferred to a fresh tube and its absorbance was measured at $532 \mathrm{~nm}^{16}$.

\section{RNA extraction and MicroRNA analysis}

Out of 44 participants, 22 participants from the active treatment group, and 21 subjects from the placebo group were randomized and evaluated regarding the microRNA. The microRNAs were analyzed at the study start and at the end of study in both the intervention and the placebo groups. The vials were retained on ice before chilled centrifugation at $3000 \mathrm{~g}$ and then frozen at $-70^{\circ} \mathrm{C}$, and no sample was melted more than twice.

Total RNA was extracted from 100 $\mu \mathrm{L}$ of serum sample using RNX-Plus reagent (Cinnagen, Iran) followed by DNase I digestion (Takara Bio Inc, Shiga, Japan) according to the user manual. The concentration and purity of extracted RNAs was evaluated by Nano drop ND-1000 spectrophotometer (Nano drop Technologies, Wilmington, DE, USA) reading at wave length 230, 260 and $280 \mathrm{~nm}$. The RNA samples were liquated in RNase free tubes and stored at $-70^{\circ} \mathrm{C}$ before use.

Complementary DNA (cDNA) was synthesized by Prime Script RT reagent kit (Takara Bio Inc, Shiga, Japan). Based on manufacturer protocol. Real-time PCR was performed using the SYBR(r) Premix Ex Taq II (Takara) and relative gene expression was calculated as $2^{-\mathrm{Ct}}$. The list of primers for specific genes has been illustrated in Table4. The miRNA Snordu6 was used as an internal control.

\section{Statistical analysis}

The results are presented as a mean \pm standard error for continuous variables, and as numbers (percentages) for categorical variables. A one-way multivariate analysis of covariance was used to control the pretest differences, followed by Dunnett's post hoc comparison for multiple comparisons between the groups and paired-sample student t tests for comparisons within each group. Finally, the data were tested for normality using the Kolmogorov-Smirnov test. Because of the non-normality of the studied variables (positively skewed distribution), logarithmic transformation was performed and homogeneity of the covariance matrix was examined using Box's M statistics. Levels of relative expression of the miRNAs were evaluated using 2$\Delta \Delta \mathrm{CT}$ method. The comparison between groups was carried out with the one-way ANOVA or KruskalWallis test was performed with a Turkey or Dunn's post-test pairwise comparison. All statistical analyses were performed using SPSS, (Version 20, SPSS Inc., Chicago, IL), and significance was set at $\mathrm{P}<0.05$.

\section{Results}

\section{Clinical and anthropometric characteristics of the study population}

Forty-four subjects with type 2 diabetes were enrolled in this study; 43 of them completed the 8 weeks' intervention. Exclusion from the study were none-compliance of study. The baseline physical and medical characterization of subjects are detailed in Table1. As indicated, no statistically significant difference was observed in terms of basic characteristics including age, gender, the body mass index (BMI), diabetes duration, basal metabolic rate (BMR), total body fat, visceral body fat, oral antidepressant drugs, and oral glucose lowering drugs between the two 8mg ASX or placebo groups.

\section{Dietary and Physical activity}

Dietary records were analyzed for total dietary energy, fat, protein, carbohydrate, and micronutrient intake among the participants with type 2 diabetes mellitus before and after the intervention. The obtained data showed that mentioned dietary parameters did not significantly change during the intervention. Moreover, the best sources of natural AST are algae, yeast, wild sockeye salmon, trout, krill, and shrimp and these were not included in the daily diet of our participants. Thus, food sources of AST were limited, and intake of other carotenoids did not differ between the groups during the study ${ }^{17}$. In addition, no significant changes were detected in the participants' physical activity through the intervention $(P=0.062)$. 


\section{Circulating miR-146a, miR-126, MDA and IL-6}

Mean plasma levels of MDA and IL-6, before and after intervention is shown in Table 2. There were significant decreases (paired $t$-test) in MDA and IL-6 levels in AST group compared with the placebo group $(P<$ 0.05). Although the comparison within the groups indicated that AST could decrease MDA level and IL-6 values over time $(P<0.05)$.

On the other hand, following the 8-week administration of AST, we observed that the expression level of miR-146-a significantly decreased in comparison to the placebo group (Fold change: -1/388) (P-value $<0.05)$. Meanwhile the increased level of the miR-126 was detected but was not statistically significant. The expression changes of miR-126 and miR-146 in the AST group before and after intervention was shown in Table3.

However, regarding placebo group, the mean expression of miR-126 was significantly decreased (Target gene up-regulated by factor: -1.233). But, circulating miR-146-a was increased in comparison to the expression of the reference gene (Target gene up-regulated by factor: 1.185). The expression changes of miR-126 and miR-146 in the placebo group before and after intervention was shown in Table3.

\section{Discussion}

This study was conducted in order to determinate the effect of $8 \mathrm{mg} /$ day AST supplementation for eight weeks on the relative expression of miR-146a and miR-126a, and also improvement of MDA and IL-6 levels.

We observed that $8 \mathrm{mg}$ of the AST supplementation significantly reduced the expression level of miR-146-a. Moreover, our results displayed that inhibition of miR-146a expression associated with a moderated release of IL-6 and MDA. However, the high expression level of miR-126 in AST-supplemented patients was not significant. This specific effect of AST on miR-146a expression behavior might be the consequence of a pro-inflammatory component of the T2D. In this regard, the results from the other studies showed that miR-146a relative expression was associated with an inflammatory marker in the T2D patients ${ }^{18}$. Also, the modulatory effect of some antioxidants on circulating MicroRNAs was examined in previous studies and suggested to consider as a novel therapeutic target in disease management in many literature ${ }^{19,20}$. Morales et al, stated the potential effect of polyphenols extracted from red wine as antioxidant increased plasma MicroRNAs in a dose-dependent manner. Also, the up regulation of MicroRNAs and attenuated the expression of pro-inflammatory cytokines was confirmed by treating cells with mango extracting rat ${ }^{21}$.

Moreover, in context to placebo group, we indicated that the expression of miR-146a was augmented during the intervention and associated with raised IL-6 and MDA which is consistent with previous studies ${ }^{18}$. Also in a study conducted $b^{22}$, a positive correlation was observed between miR-146a levels and concentration of inflammatory mediators, whereas, elevated miR-146a inhibited the production of IL- 6 and IL-21 in T cells. These results in consistent with our obtained results. In addition, several studies revealed that miR-146a, is overexpressed in type 2 diabetes patients compared with healthy controls ${ }^{8,22}$, which it described serum miRNAs as a explore DM biomarkers. Furthermore, the results from the present study were in line with previous report and show that high hyperglycemia significantly reduced miR-126 release, the most abundant miRNAs in endothelial apoptotic bodies, from endothelial cells ${ }^{23}$. And loss of miR- 126 has been proposed as a predictor of type 2 diabetes onset ${ }^{24}$.

However, this is the first study that assesses the effect of AST on circulating miRNAs and we have a lack of experiments comparing the potential effect of AST supplementation on diabetes patients with other studies. In addition, short study duration may affect the process of genetic outcome and we could not demonstrate antioxidant agents of AST on modulating plasma miR-126 levels through reducing inflammatory pathways. So further larger sample size studies are urgent to achieve this goal and our findings should be considered preliminary and the requirement for further studies is indicated.

\section{Conclusion}

In summary, our results introduced $8 \mathrm{mg} /$ day of AST supplementation for 8 weeks as a new agent for 
decreasing the circulating MDA, IL-6 and relative expression of MiR-146a. However, further larger sample size studies are needed to confirm these outcomes.

\section{Acknowledgment}

This study is part of the registered protocol in the Iranian Registry of Clinical Trials (IRCT2015081623645N1) which was approved by the Research Ethics Committee of Ahvaz University of Medical Sciences.

\section{Conflict of interest}

None of the authors has any potential financial conflict of interest related to this manuscript.

\section{Funding source}

This research did not receive any specific grant from funding agencies in the public, commercial, or not-forprofit sectors

\section{References}

1. Association AD. Diagnosis and classification of diabetes mellitus.Diabetes care. 2013;36(Supplement 1):S67-S74.

2. Halban PA, Polonsky KS, Bowden DW, et al. $\beta$-cell failure in type 2 diabetes: postulated mechanisms and prospects for prevention and treatment. The Journal of Clinical Endocrinology 83 Metabolism.2014;99(6):1983-1992.

3. Koloverou E, Panagiotakos DB. Inflammation: a new player in the link between Mediterranean diet and diabetes mellitus: a review.Current Nutrition Reports. 2017;6(3):247-256.

4. Donath MY, Størling J, Maedler K, Mandrup-Poulsen T. Inflammatory mediators and islet $\beta$-cell failure: a link between type 1 and type 2 diabetes. Journal of molecular medicine. 2003;81(8):455-470.

5. Gareri C, De Rosa S, Indolfi C. MicroRNAs for restenosis and thrombosis after vascular injury. Circulation research.2016;118(7):1170-1184.

6. Hashimoto N, Tanaka T. Role of miRNAs in the pathogenesis and susceptibility of diabetes mellitus. Journal of human genetics.2017;62(2):141.

7. Rottiers V, Näär AM. MicroRNAs in metabolism and metabolic disorders. Nature reviews Molecular cell biology. 2012;13(4):239.

8. Rong Y, Bao W, Shan Z, et al. Increased microRNA-146a levels in plasma of patients with newly diagnosed type 2 diabetes mellitus. PloS one. 2013;8(9):e73272.

9. Liu Y, Gao G, Yang C, et al. The role of circulating microRNA-126 (miR-126): a novel biomarker for screening prediabetes and newly diagnosed type 2 diabetes mellitus. International journal of molecular sciences. 2014;15(6):10567-10577.

10. Osmai M, Osmai Y, Bang-Berthelsen $\mathrm{CH}$, et al. MicroRNAs as regulators of beta-cell function and dysfunction. Diabetes/metabolism research and reviews. 2016;32(4):334-349.

11. Al-Muhtaresh H, Al-Kafaji G. Evaluation of two-diabetes related microRNAs suitability as earlier blood biomarkers for detecting prediabetes and type 2 diabetes mellitus. Journal of clinical medicine. 2018;7(2):12.

12. Babashah S, Bakhshinejad B, Birgani MT, Pakravan K, Cho WC. Regulation of microRNAs by phytochemicals: A promising strategy for cancer chemoprevention. Current cancer drug targets.2018;18(7):640651.

13. Meydani M, Azzi A. Dietary antioxidants and bioflavonoids in atherosclerosis and angiogenesis. Nutrigenomics and Proteomics in Health and Disease: Towards a systems-level understanding of gene-diet interactions. 2017:125-142. 
14. Sila A, Ghlissi Z, Kamoun Z, et al. Astaxanthin from shrimp by-products ameliorates nephropathy in diabetic rats. European journal of nutrition. 2015;54(2):301-307.

15. Roohbakhsh A, Karimi G, Iranshahi M. Carotenoids in the treatment of diabetes mellitus and its complications: A mechanistic review.Biomedicine \& Pharmacotherapy. 2017;91:31-42.

16. Hosseinzadeh H, Sadeghnia HR, Ziaee T, Danaee A. Protective effect of aqueous saffron extract (Crocus sativus L.) and crocin, its active constituent, on renal ischemia-reperfusion-induced oxidative damage in rats. J Pharm Pharm Sci. 2005;8(3):387-393.

17. Mashhadi NS, Zakerkish M, Mohammadiasl J, Zarei M, Mohammadshahi M, Haghighizadeh MH. Astaxanthin improves glucose metabolism and reduces blood pressure in patients with type 2 diabetes mellitus. Asia Pacific journal of clinical nutrition. 2018;27(2):341.

18. Garcia-Jacobo RE, Uresti-Rivera EE, Portales-Perez DP, et al. Circulating miR-146a, miR-34a and miR-375 in type 2 diabetes patients, pre-diabetic and normal-glycaemic individuals in relation to $\beta$-cell function, insulin resistance and metabolic parameters. Clinical and Experimental Pharmacology and Physiology. 2019;46(12):1092-1100.

19. Khatir SA, Bayatian A, Barzegari A, et al. Saffron (Crocus sativus L.) Supplements Modulate Circulating MicroRNA (miR-21) in Atherosclerosis Patients; A Randomized, Double-Blind, Placebo-Controlled Trial. Iranian Red Crescent Medical Journal. 2018;20(10).

20. Corrêa TA, Rogero MM. Polyphenols regulating microRNAs and inflammation biomarkers in obesity. Nutrition. 2019;59:150-157.

21. Angel-Morales G, Noratto G, Mertens-Talcott S. Red wine polyphenolics reduce the expression of inflammation markers in human colon-derived CCD-18Co myofibroblast cells: potential role of microRNA-126. Food E function. 2012;3(7):745-752.

22. Saba R, Gushue S, Huzarewich RL, et al. MicroRNA 146a (miR-146a) is over-expressed during prion disease and modulates the innate immune response and the microglial activation state. PloS one.2012;7(2):e30832.

23. Zampetaki A, Kiechl S, Drozdov I, et al. Plasma microRNA profiling reveals loss of endothelial miR-126 and other microRNAs in type 2 diabetes. Circulation research. 2010;107(6):810-817.

24. Zhang T, Li L, Shang Q, Lv C, Wang C, Su B. Circulating miR-126 is a potential biomarker to predict the onset of type 2 diabetes mellitus in susceptible individuals. Biochemical and biophysical research communications. 2015;463(1):60-63.

\section{Hosted file}

figure.docx available at https://authorea.com/users/355778/articles/481411-the-effects-ofastaxanthin-supplementation-on-the-expression-of-mir-146a-and-mir-126-oxidative-stressand-inflammation-in-patients-with-type-2-diabetes-mellitus-a-randomized-double-blindplacebo-controlled-clinical-trial

\section{Hosted file}

tabels.docx available at https://authorea.com/users/355778/articles/481411-the-effects-ofastaxanthin-supplementation-on-the-expression-of-mir-146a-and-mir-126-oxidative-stressand-inflammation-in-patients-with-type-2-diabetes-mellitus-a-randomized-double-blindplacebo-controlled-clinical-trial 